\title{
ON THE GENERALIZED DRIFT SKOROKHOD PROBLEM IN ONE DIMENSION
}

\author{
JOSH REED, ${ }^{*}$ New York University \\ AMY WARD ** AND \\ DONGYUAN ZHAN, ${ }^{* *}$ University of Southern California
}

\begin{abstract}
We show how to write the solution to the generalized drift Skorokhod problem in onedimension in terms of the supremum of the solution of a tractable unrestricted integral equation (that is, an integral equation with no boundaries). As an application of our result, we equate the transient distribution of a reflected Ornstein-Uhlenbeck (OU) process to the first hitting time distribution of an OU process (that is not reflected). Then, we use this relationship to approximate the transient distribution of the GI/GI/1 + GI queue in conventional heavy traffic and the $\mathrm{M} / \mathrm{M} / N / N$ queue in a many-server heavy traffic regime.
\end{abstract}

Keywords: Skorokhod map; reflected Ornstein-Uhlenbeck process; abandonment; queueing

2010 Mathematics Subject Classification: Primary 90B22

Secondary 90B15; 60G17; 60J60

\section{Introduction}

The Skorokhod problem was originally introduced by Skorokhod [15] in order to study continuous solutions to stochastic differential equations with a reflecting boundary at zero.

Definition 1.1. (Skorokhod problem.) Given a process $X \in D([0, \infty), \mathbb{R})$, we say that the pair of processes $(Z, L) \in D^{2}([0, \infty), \mathbb{R})$ satisfy the Skorokhod problem for $X$ if the following four conditions are satisfied:

1. $Z(t)=X(t)+L(t)$ for $t \geq 0$,

2. $Z(t) \geq 0$ for $t \geq 0$,

3. $L$ is nondecreasing with $L(0-)=0$,

4. $\int_{0}^{\infty} \mathbf{1}\{Z(t)>0\} \mathrm{d} L(t)=0$.

It is well known that, for each $X \in D([0, \infty), \mathbb{R})$, the unique solution $(Z, L)=(\Phi(X)$, $\Psi(X))$ to the Skorokhod problem is

$$
Z(t)=X(t)+\sup _{0 \leq s \leq t}-X(s) \vee 0 \quad \text { and } \quad L(t)=\sup _{0 \leq s \leq t}-X(s) \vee 0 .
$$

Received 7 January 2011; revision received 11 July 2012.

* Postal address: Stern School of Business, New York University, New York, NY 10012, USA.

Email address: jreed@stern.nyu.edu

** Postal address: Marshall School of Business, University of Southern California, Los Angeles, CA 90089-0809, USA. 
In subsequent papers, the Skorokhod problem has been extended to multiple dimensions and also to include both smooth and nonsmooth domains (see, for example, [4], [6], [8], [13], and [17]), although we do not treat such cases in the present paper. There is a useful integral representation of the one-dimensional Skorokhod problem solution (see [2]). There is also an explicit solution to the (one-dimensional) Skorokhod problem when there is an upper boundary (see [9] and [10])) and to the (one-dimensional) Skorokhod problem in a time-dependent interval (see [3]).

In this paper we study a generalization of the one-dimensional Skorokhod problem that incorporates a state-dependent drift.

Definition 1.2. (Generalized drift Skorokhod problem in one dimension.) Given a process $X \in D([0, \infty), \mathbb{R})$ with $X(0)=0$ and a Lipschitz continuous function $f: \mathbb{R}_{+} \rightarrow \mathbb{R}$, we say that the pair of processes $(Z, L) \in D^{2}([0, \infty), \mathbb{R})$ satisfy the Skorokhod problem for $X$ with state-dependent drift function $f$ if the following four conditions are satisfied:

1. $Z(t)=X(t)-\int_{0}^{t} f(Z(s)) \mathrm{d} s+L(t)$ for $t \geq 0$,

2. $Z(t) \geq 0$ for $t \geq 0$,

3. $L$ is nondecreasing with $L(0-)=0$,

4. $\int_{0}^{\infty} \mathbf{1}\{Z(t)>0\} \mathrm{d} L(t)=0$.

The unique solution to the generalized drift Skorokhod problem in one dimension can be written in terms of the solution to the Skorokhod problem following a standard construction; see, for example, [22]. Specifically, set

$$
(Z, L)=(\Phi(\mathcal{M}(X)), \Psi(\mathcal{M}(X)))
$$

for $\mathcal{M}: D([0, \infty), \mathbb{R}) \rightarrow D([0, \infty), \mathbb{R})$, the mapping that sets $\mathcal{M}(X)=V$ for $V$ that solves the integral equation

$$
V(t)=X(t)-\int_{0}^{t} f(\Phi(V)(s)) \mathrm{d} s \quad \text { for all } t \geq 0 .
$$

Note that, since $f: \mathbb{R}_{+} \rightarrow \mathbb{R}$ is a Lipschitz continuous function, a standard Picard iteration shows that there exists a unique solution to (1.2). The fact that $(\Phi(\mathcal{M}(X)), \Psi(\mathcal{M}(X)))$ solves the Skorokhod problem for $\mathcal{M}(X)$ (and so satisfies conditions 1-4 of Definition 1.1) shows that conditions 1-4 of Definition 1.2 are satisfied. The uniqueness of representation (1.1) follows from the uniqueness of the mappings $\mathcal{M}$ and $(\Phi, \Psi)$.

Next, we observe that the solution $Z$ can be represented in terms of an unrestricted integral equation (that is, an integral equation with no boundaries). Specifically, note that, from (1.2),

$$
V(t)-V(s)=X(t)-X(s)-\int_{s}^{t} f(\Phi(V)(u)) \mathrm{d} u .
$$

Since, when $X(0)=0$,

$$
\Phi(V)(u)=\sup _{0 \leq r \leq u} V(u)-V(r)
$$

if we define

$$
R(s, t)=V(t)-V(s)
$$


then

$$
R(s, t)=X(t)-X(s)-\int_{s}^{t} f\left(\sup _{0 \leq r \leq u} R(r, u)\right) \mathrm{d} u .
$$

Finally, it follows from (1.1) and the above displays that

$$
Z(t)=\sup _{0 \leq s \leq t} R(s, t) .
$$

However, the integral equation (1.3) is not tractable.

In this paper we establish how to represent $Z$ in terms of the solution to a tractable unrestricted integral equation. Specifically, we establish that

$$
Z(t)=\sup _{0 \leq s \leq t} Z_{s}(t-s), \quad t \geq 0,
$$

for $Z_{s}=\left\{Z_{S}(t), t \geq 0\right\}$ that solves

$$
Z_{s}(t)=X(s+t)-X(s)-\int_{0}^{t} f_{\mathrm{e}}\left(Z_{s}(u)\right) \mathrm{d} u,
$$

where $f_{\mathrm{e}}: \mathbb{R} \rightarrow \mathbb{R}$ is any extension of $f: \mathbb{R}_{+} \rightarrow \mathbb{R}$ that preserves the Lipschitz continuity of $f$. For one example, let $f_{\mathrm{e}}(x)=f(0)$ if $x<0$ and $f_{\mathrm{e}}(x)=f(x)$ if $x \geq 0$. It is interesting to observe that it follows from (1.4) and (1.5) that

$$
\sup _{0 \leq s \leq t} R(s, t)=\sup _{0 \leq s \leq t} Z_{s}(t-s) .
$$

As an application of representation (1.5), we show how to use (1.5) to write the transient distribution of a reflected Ornstein-Uhlenbeck (OU) process in terms of the first hitting time distribution of an unreflected OU process, which additionally yields a uniform integrability result for reflected OU processes. Such a result can also be derived using duality theory (see, for example, [5] and [14]); however, the proof methodology is much different, because there is no sample path representation that is equivalent to (1.5) in either [5] or [14]. Because the reflected OU process has been shown to approximate the GI/GI/1+GI and $\mathrm{M} / \mathrm{M} / N / N$ queues (see [20] and [16]), we see that the transient distribution of the number-in-system process for the $\mathrm{GI} / \mathrm{GI} / 1+\mathrm{GI}$ and $\mathrm{M} / \mathrm{M} / N / N$ queues can be approximated by the first hitting time distribution of an OU process (that is not reflected).

The remainder of this paper is organized as follows. In Section 2 we prove (1.5). In Section 3 we apply (1.5) in the context of a reflected OU process. In Section 4 we perform simulation studies that support approximating the transient distribution of the number-in-system process for the GI/GI/ $1+\mathrm{GI}$ and $\mathrm{M} / \mathrm{M} / N / N$ queues with the first hitting time distribution of an $\mathrm{OU}$ process (that is not reflected).

\section{The generalized drift Skorokhod problem solution (in one dimension)}

In this section we establish (1.5).

Theorem 2.1. Let $(Z, L)$ be the unique solution to the generalized Skorokhod problem for $X$ with $X(0)=0$, and with state-dependent drift function $f$ that is Lipschitz continuous. For each $s \geq 0$, let $Z_{s}$ be defined as in (1.6). Then, for each $t \geq 0$,

$$
Z(t)=\sup _{0 \leq s \leq t} Z_{s}(t-s)
$$


Proof. We first claim that, for each $0 \leq s \leq t$,

$$
Z_{s}(t-s) \leq Z(t) \text {. }
$$

To see this, first recall from (1.6) that $Z_{s}(t-s)$ is the solution to the equation

$$
Z_{s}(u)=X(s+u)-X(s)-\int_{0}^{u} f_{\mathrm{e}}\left(Z_{s}(v)\right) \mathrm{d} v,
$$

evaluated at the point $u=t-s$, where $f_{\mathrm{e}}: \mathbb{R} \mapsto \mathbb{R}$ is an arbitrary Lipschitz extension of $f: \mathbb{R}_{+} \mapsto \mathbb{R}$. Next, it is straightforward to see from condition 1 of Definition 1.1 that $Z(t)$ is the unique solution to the equation

$$
Z(s+u)=Z(s)+(X(s+u)-X(s)+L(s+u)-L(s))-\int_{0}^{u} f_{\mathrm{e}}(Z(s+v)) \mathrm{d} v
$$

for $u \geq 0$, also evaluated at the point $u=t-s$ (note that in (2.2) we have replaced $f$ by $f_{\mathrm{e}}$ ). Subtracting (2.1) from (2.2) we therefore obtain

$$
\left(Z(s+u)-Z_{s}(u)\right)=Z(s)+L(s+u)-L(s)-\int_{0}^{u}\left(f_{\mathrm{e}}(Z(s+v))-f_{\mathrm{e}}\left(Z_{s}(v)\right)\right) \mathrm{d} v
$$

for $u \geq 0$. Note also that by the Lipschitz continuity of $f_{\mathrm{e}}$ we have, for some constant $K>0$,

$$
\left(Z(s+u)-Z_{s}(u)\right) \geq Z(s)+L(s+u)-L(s)-K \int_{0}^{u}\left|Z(s+v)-Z_{s}(v)\right| \mathrm{d} v
$$

for $u \geq 0$. Now consider the solution $W_{s}=\left\{W_{s}(u), u \geq 0\right\}$ to the ordinary differential equation

$$
W_{s}(u)=Z(s)+L(s+u)-L(s)-K \int_{0}^{u}\left|W_{s}(v)\right| \mathrm{d} v, \quad u \geq 0 .
$$

We claim that

$$
W_{s}(u)=Z(s) \mathrm{e}^{-K u}+\int_{0}^{u} \mathrm{e}^{K(v-u)} \mathrm{d} L(s+v), \quad u \geq 0 .
$$

This may be verified by noting that $W_{s}(u) \geq 0$ for $u \geq 0$, since $Z(s) \geq 0$ and $L$ is a nondecreasing function. Subtracting (2.4) from (2.3) and using Gronwall's inequality, it follows that $Z(s+u)-Z_{s}(u) \geq W_{s}(u) \geq 0$, and so $Z(s+u) \geq Z_{s}(u)$, which, evaluating at $u=t-s$, yields $Z_{s}(t-s) \leq Z(t)$, our desired result. We have therefore shown that

$$
Z(t) \geq \sup _{0 \leq s \leq t}\left\{Z_{s}(t-s)\right\}
$$

It now remains to reverse the direction of the inequality in (2.5). In order to do so, it suffices to show that there exists at least one point $s^{\star}$ such that $Z_{s^{\star}}\left(t-s^{\star}\right)=Z(t)$. Let $s^{\star}=\sup \{s \leq t: Z(s)=0\}$ be the last time at which the process $Z$ hit zero. Note that $s^{\star}$ is well defined since $Z(0)=0$. Also, note that $L(s)=L\left(s^{\star}\right)$ for $s \geq s^{\star}$. Thus, by (2.2) we have

$$
Z\left(s^{\star}+u\right)=X\left(s^{\star}+u\right)-X\left(s^{\star}\right)-\int_{0}^{u} f_{\mathrm{e}}\left(Z\left(s^{\star}+v\right)\right) \mathrm{d} v, \quad u \geq 0,
$$

and so, $Z\left(s^{\star}+u\right)=Z_{s^{\star}}(u)$ for $0 \leq u \leq t-s^{\star}$, and, in particular, $Z(t)=Z_{s^{\star}}\left(t-s^{\star}\right)$, which completes the proof. 


\section{Reflected OU processes}

In this section we let the process $X$ in the definition of the generalized Skorokhod problem be a Brownian motion with constant drift $\theta$ and infinitesimal variance $\sigma^{2}$ defined on a suitable probability space $(\Omega, \mathcal{F}, \mathbb{P})$. We also set $f(x)=\gamma x$ for $x \geq 0$ and some $\gamma \in \mathbb{R}$. The resulting process $Z$, defined sample pathwise as the solution to the generalized Skorokhod problem for $X$ and $f$, is referred to as a $(\sigma, \theta, \gamma)$ reflected OU process, which has initial condition $Z(0)=0$. It is immediate that the following definition of a reflected OU process is equivalent to the prescription given above.

Definition 3.1. (Reflected OU process.) Let $B=\{B(t), t \geq 0\}$ be a standard Brownian motion defined on a probability space $(\Omega, \mathcal{F}, \mathbb{P})$, and let $\sigma>0$, and $\theta, \gamma \in \mathbb{R}$. We say that the process $Z$ is a $(\sigma, \theta, \gamma)$ reflected OU process if the following four conditions are satisfied $\mathbb{P}$-almost-surely:

1. $Z(t)=\sigma B(t)+\theta t-\gamma \int_{0}^{t} Z(s) \mathrm{d} s+L(t)$ for $t \geq 0$,

2. $Z(t) \geq 0$ for $t \geq 0$,

3. $L$ is nondecreasing with $L(0-)=0$,

4. $\int_{0}^{\infty} \mathbf{1}\{Z(t)>0\} \mathrm{d} L(t)=0$.

Now, for each $s \geq 0$, recall from (1.6) the definition of the associated unreflected processes

$$
Z_{s}(u)=(\sigma B(s+u)+\theta(s+u))-(\sigma B(s)+\theta s)-\gamma \int_{0}^{u} Z_{s}(v) \mathrm{d} v
$$

for $u \geq 0$, where here we have set $X(t)=\sigma B(t)+\theta t$, and we take the natural extension $f_{\mathrm{e}}(x)=\gamma x$ for $x \in \mathbb{R}$. For clarity of exposition in the sequel, we now hold $t \geq 0$ fixed and define the new process

$$
Y_{t}(u)=Z_{t-u}(u), \quad 0 \leq u \leq t .
$$

Since $\left\{Y_{t}(u), 0 \leq u \leq t\right\}$ is just the process $\left\{Z_{s}(t-s), 0 \leq s \leq t\right\}$ run backwards in time, it follows that

$$
\sup _{0 \leq u \leq t}\left\{Y_{t}(u)\right\}=\sup _{0 \leq s \leq t}\left\{Z_{s}(t-s)\right\}
$$

and so it follows from Theorem 2.1 that if $Z$ is a $(\sigma, \theta, \gamma)$ reflected OU process then

$$
Z(t)=\sup _{0 \leq u \leq t}\left\{Y_{t}(u)\right\}
$$

In preparation for our next result, we now say that a process $X$ is a $(\sigma, \theta, \gamma)$ OU process starting from $X(0)$ (note the absence of reflection here) if it is the unique strong solution to the stochastic differential equation

$$
X(t)=X(0)+\sigma B(t)+\theta t-\int_{0}^{t} \gamma X(s) \mathrm{d} s
$$

for $t \geq 0$, where $B$ is a standard Brownian motion. We then make the following claim regarding the process $\left\{Y_{t}(u), 0 \leq u \leq t\right\}$.

Proposition 3.1. The process $\left\{\mathrm{e}^{\gamma u} Y_{t}(u), 0 \leq u \leq t\right\}$ is equal in distribution to a $(\sigma, \theta,-\gamma)$ OU process on $[0, t]$ which starts from 0 . 
Proof. First note that, since $X(t)=\sigma B(t)+\theta t$ is a Brownian motion with infinitesimal variance $\sigma^{2}$ and constant drift $\theta$, it follows that, for each $s \geq 0$, the process $X_{s}=\{X(s+t)-$ $X(s), t \geq 0\}$ is also Brownian motion with the same parameters and so, for each $s \geq 0$, the process $\bar{Z}_{s}=\left\{Z_{s}(u), u \geq 0\right\}$ is an OU process whose explicit solution is given by

$$
Z_{S}(u)=\frac{\theta}{\gamma}\left(1-\mathrm{e}^{-\gamma u}\right)+\int_{0}^{u} \sigma \mathrm{e}^{\gamma(v-u)} \mathrm{d} B_{S}(v), \quad u \geq 0,
$$

where $B_{s}=\{B(s+t)-B(s), t \geq 0\}$.

Setting $Y_{t}(u)=Z_{t-u}(u)$, it therefore follows that

$$
Y_{t}(u)=\frac{\theta}{\gamma}\left(1-\mathrm{e}^{-\gamma u}\right)+\int_{0}^{u} \sigma \mathrm{e}^{\gamma(v-u)} \mathrm{d} B_{t-u}(v) .
$$

However, since $\mathrm{d} B_{t-u}(v)=\mathrm{d} B(t-u+v)$, the change with respect to $v$, it follows that making the change of variable $\zeta=u-v$, the above becomes

$$
\begin{aligned}
Y_{t}(u) & =\frac{\theta}{\gamma}\left(1-\mathrm{e}^{-\gamma u}\right)+\int_{0}^{u} \sigma \mathrm{e}^{\gamma(v-u)} \mathrm{d} B(t-u+v) \\
& =\frac{\theta}{\gamma}\left(1-\mathrm{e}^{-\gamma u}\right)+\int_{u}^{0} \sigma \mathrm{e}^{-\gamma \zeta} \mathrm{d} B(t-\zeta) \\
& =\frac{\theta}{\gamma}\left(1-\mathrm{e}^{-\gamma u}\right)-\int_{0}^{u} \sigma \mathrm{e}^{-\gamma \zeta} \mathrm{d} B(t-\zeta) .
\end{aligned}
$$

However, it is clear that the above, as a process, is also equal in distribution to

$$
\frac{\theta}{\gamma}\left(1-\mathrm{e}^{-\gamma u}\right)+\int_{0}^{u} \sigma \mathrm{e}^{-\gamma t} \mathrm{~d} B(t), \quad u \geq 0 .
$$

Multiplying both sides of the above by $\mathrm{e}^{\gamma u}$, we then obtain

$$
\mathrm{e}^{\gamma u} Y_{t}(u)=-\frac{\theta}{\gamma}\left(1-\mathrm{e}^{\gamma u}\right)+\int_{0}^{u} \sigma \mathrm{e}^{-\gamma(t-u)} \mathrm{d} B(t),
$$

which is just an OU process on $[0, t]$ with infinitesimal variance $\sigma^{2}$, constant drift $\theta$, and linear drift $-\gamma$.

The following is our main result of this section, relating the distribution of the supremum appearing in (3.1) to the first hitting distribution of an OU process. Let

$$
\sigma_{x}=\inf \{t \geq 0: U(t)=x\},
$$

where $U=\{U(t), t \geq 0\}$ is an OU process with parameters $(\sigma,-\gamma x+\theta,-\gamma)$ and started from 0 . In other words, $\sigma_{x}$ is the first hitting time of $x$ by $U$. We then have the following proposition.

Proposition 3.2. For each $t \geq 0$,

$$
\mathbb{P}(Z(t) \geq x)=\mathbb{P}\left(\sigma_{x} \leq t\right) .
$$


Proof. Note that, for each $x \geq 0$,

$$
\begin{aligned}
\left\{\sup _{0 \leq u \leq t} Y_{t}(u) \geq x\right\} & =\left\{\inf \left\{u: Y_{t}(u) \geq x\right\} \leq t\right\} \\
& =\left\{\inf \left\{u: \mathrm{e}^{\gamma u} Y_{t}(u) \geq \mathrm{e}^{\gamma u} x\right\} \leq t\right\} \\
& =\left\{\inf \left\{u: x\left(1-\mathrm{e}^{\gamma u}\right)+\mathrm{e}^{\gamma u} Y_{t}(u) \geq x\right\} \leq t\right\} .
\end{aligned}
$$

Now, by Proposition 3.1, $\left\{x\left(1-\mathrm{e}^{\gamma u}\right)+\mathrm{e}^{\gamma u} Y_{t}(u), u \geq 0\right\}$ is simply an OU process with infinitesimal variance $\sigma^{2}$, constant drift $-\gamma x+\theta$, and linear drift $-\gamma$. The result then follows immediately.

Sigman and Ryan [14] established an equivalent result to Proposition 3.2; however, their proof methodology is much different. In particular, Sigman and Ryan related the transient distribution of any continuous-time, real-valued stochastic process that can be defined recursively (either explicitly in discrete time or implicitly in continuous time, through the use of an integral equation) to the ruin time of a dual risk process. There is no result in [14] that is equivalent to Theorem 2.1, which is the basis for our proof of Proposition 3.2.

\subsection{Computing the first hitting time}

In order to use Proposition 3.2 to compute $\mathbb{P}(Z(t) \geq x)$, it is necessary that the distribution of $\sigma_{x}$ is known. Fortunately, there are various results in the literature available for computing the first hitting time distributions of OU processes. Linetsky [11] provided a spectral expansion for the first hitting time of OU processes and the results of Alili et al. [1] provide three different means to compute various probabilities associated with this hitting time. In what follows, we use the results in [1].

Let $p_{x_{0} \rightarrow x}^{(\sigma, \theta, \gamma)}$ denote the density of the distribution of $\sigma_{x}$ for a $(\sigma, \theta, \gamma)$ OU process, so that we may write

$$
\mathbb{P}\left(\sigma_{x} \leq t\right)=\int_{0}^{t} p_{x_{0} \rightarrow x}^{(\sigma, \theta, \gamma)}(s) \mathrm{d} s, \quad t \geq 0 .
$$

Alili et al. [1] showed how to calculate $p_{x_{0} \rightarrow x}^{(1,0, \gamma)}$ when $\gamma>0$. Since we are interested in the more general case, we first express $p_{x_{0} \rightarrow x}^{(\sigma, \theta, \gamma)}$ in terms of $p_{x_{0} \rightarrow x}^{(1,0, \gamma)}$. In order to do this, note that, since a $(\sigma, \theta, \gamma)$ OU process starting from $x_{0}$ has the same distribution as a $(1, \theta / \sigma, \gamma) \mathrm{OU}$ process starting from $x_{0} / \sigma$, it follows that

$$
p_{x_{0} \rightarrow x}^{(\sigma, \theta, \gamma)}(t)=p_{x_{0} / \sigma \rightarrow x / \sigma}^{(1, \theta / \sigma, \gamma)}(t), \quad t \geq 0
$$

Next, Remark 2.5 of [1] shows that

$$
p_{x_{0} / \sigma \rightarrow x / \sigma}^{(1, \theta / \sigma, \gamma)}(t)=p_{x_{0} / \sigma-\theta / \sigma \gamma \rightarrow x / \sigma-\theta / \sigma \gamma}^{(1,0, \gamma)}(t), \quad t \geq 0 .
$$

When $x-\theta / \gamma=0$, the above expression may be immediately evaluated because

$$
p_{\zeta \rightarrow 0}^{(1,0, \gamma)}(t)=\frac{|\zeta|}{\sqrt{2 \pi}}\left(\frac{\lambda}{\sinh (\lambda t)}\right)^{3 / 2} \exp \left(-\frac{\lambda \zeta^{2} \mathrm{e}^{-\lambda t}}{2 \sinh (\lambda t)}+\frac{\lambda t}{2}\right)
$$

as is found in [12] and reproduced in Equation (2.8) of [1]. Otherwise, when $x-\theta / \gamma \neq 0$, one must appeal to one of the three representations in [1] (one that hinges on an eigenvalue expansion, one that is an integral representation, and one that is given in terms of a functional of a three-dimensional Bessel bridge) in order to compute $\mathbb{P}\left(\sigma_{x} \leq t\right)$. 
To compute the transient distribution of the $(\sigma, \theta, \gamma)$ reflected OU process $Z$, we first apply Proposition 3.2, and then use the distributional equalities (3.2) and (3.3) as follows:

$$
\begin{aligned}
\mathbb{P}(Z(t) \geq x) & =\mathbb{P}\left(\sigma_{x} \leq t\right) \\
& =\int_{0}^{t} p_{0 \rightarrow x}^{(\sigma,-\gamma x+\theta,-\gamma)}(s) \mathrm{d} s \\
& =\int_{0}^{t} P_{0 \rightarrow x / \sigma}^{(1,(-\gamma x+\theta) / \sigma,-\gamma)}(s) \mathrm{d} s \\
& =\int_{0}^{t} p_{\theta / \sigma \gamma-x / \sigma \rightarrow \theta / \sigma \gamma}^{(1,0,-\gamma)}(s) \mathrm{d} s .
\end{aligned}
$$

We double check the calculation (3.5) by recalling that it also follows [14]. Specifically, Proposition 4.3 of [14] establishes that

$$
\mathbb{P}(Z(t) \geq x)=\mathbb{P}\left(\sigma^{R} \leq t\right),
$$

where $\sigma^{R}$ is the first time a $(\sigma,-\theta,-\gamma)$ OU process with initial point $x>0$ becomes negative. To see that (3.5) and (3.6) are equivalent, first observe that

$$
\begin{aligned}
\mathbb{P}\left(\sigma^{R} \leq t\right) & =\int_{0}^{t} p_{x \rightarrow 0}^{(\sigma,-\theta,-\gamma)}(s) \mathrm{d} s \\
& =\int_{0}^{t} p_{x / \sigma \rightarrow 0}^{(1,-\theta / \sigma,-\gamma)}(s) \mathrm{d} s \\
& =\int_{0}^{t} p_{x / \sigma-\theta / \sigma \gamma \rightarrow-\theta / \sigma \gamma}^{(1,0,-\gamma)}(s) \mathrm{d} s,
\end{aligned}
$$

where the second and third equalities follow from (3.2) and (3.3). Then, since symmetry implies that

$$
p_{\theta / \sigma \gamma-x / \sigma \rightarrow \theta / \sigma \gamma}^{(1,0,-\gamma)}(s)=p_{x / \sigma-\theta / \sigma \gamma \rightarrow-\theta / \sigma \gamma}^{(1,0,-\gamma)}(s)
$$

we conclude that $\mathbb{P}\left(\sigma_{x} \leq t\right)=\mathbb{P}\left(\sigma^{R} \leq t\right)$.

\subsection{Uniform integrability}

It is well known (see, for example, Proposition 1 of [19]) that if $\gamma>0$ then, for a $(\sigma, \theta, \gamma)$ reflected OU process, $Z(t) \Rightarrow Z(\infty)$ as $t \rightarrow \infty$, where $Z(\infty)$ is a normal random variable with mean $\theta / \gamma$ and variance $\sigma^{2} /(2 \gamma)$ conditioned to be positive. We now show that the sequence of random variables $\{Z(t), t \geq 0\}$ is uniformly integrable as well.

Proposition 3.3. If $\gamma>0$ then, for a $(\sigma, \theta, \gamma)$ reflected $O U$ process started at the origin, the sequence of random variables $\{Z(t), t \geq 0\}$ is uniformly integrable.

Proof. First note that, without loss of generality, we may assume that $\sigma=1$ since otherwise we may rescale. Now recall that, by Proposition 3.2, it follows that $\mathbb{P}(Z(t) \geq x)=\mathbb{P}\left(\sigma_{x} \leq t\right)$, where $\sigma_{x}=\inf \left\{t \geq 0: U_{t}=x\right\}$ and $U_{t}$ is an OU process with parameters $(1,-\gamma x+\theta,-\gamma)$ started from 0 . Hence, is suffices to show that there exists a function $g$ integrable on $\mathbb{R}^{+}$such that $\mathbb{P}\left(\sigma_{x} \leq t\right) \leq g(x)$ for all $x, t \geq 0$.

Next, it follows from (3.5) that $\mathbb{P}\left(\sigma_{x} \leq t\right)=\int_{0}^{t} p_{\theta / \gamma-x \rightarrow \theta / \gamma}^{(1,0,-\gamma)}(s) \mathrm{d} s$. Remark 2.4 of [1] shows that

$$
p_{\theta / \gamma-x \rightarrow \theta / \gamma}^{(1,0,-\gamma)}(s)=\exp \left(\gamma\left(\frac{\theta^{2}}{\gamma^{2}}-\left(\frac{\theta}{\gamma}-x\right)^{2}-s\right)\right) p_{\theta / \gamma-x \rightarrow \theta / \gamma}^{(1,0, \gamma)}(s) .
$$


(We note that there is a missing negative sign in the display appearing in Remark 2.4 of [1]; specifically, the correct equation is $p_{x \rightarrow a}^{(\lambda)}(t)=\exp \left(-\lambda\left(a^{2}-x^{2}-t\right)\right) p_{x \rightarrow a}^{(-\lambda)}(t)$.) Hence,

$$
\begin{aligned}
\mathbb{P}\left(\sigma_{x} \leq t\right) & =\exp \left(-\gamma\left(x^{2}-2 \frac{\theta}{\gamma} x\right)\right) \int_{0}^{t} \exp (-\gamma s) p_{\theta / \gamma-x \rightarrow \theta / \gamma}^{(1,0, \gamma)}(s) \mathrm{d} s \\
& \leq \exp \left(-\gamma\left(x^{2}-2 \frac{\theta}{\gamma} x\right)\right),
\end{aligned}
$$

where the last inequality follows since

$$
\int_{0}^{\infty} p_{\theta / \gamma-x \rightarrow \theta / \gamma}^{(1,0, \gamma)}(s) \mathrm{d} s=1
$$

Finally, since, for $\gamma>0$,

$$
\int_{0}^{\infty} \exp \left(-\gamma\left(x^{2}-2 \frac{\theta}{\gamma} x\right)\right) \mathrm{d} x<\infty
$$

the proof is complete.

\section{Approximating the transient distribution of the GI/GI/1 + GI and $M / M / N / N$ queues}

In this section we perform simulation studies that support using the first hitting time distribution of an OU process (that is not reflected) to approximate the transient distribution of the number-in-system process for the GI/GI/1 + GI queue (Section 4.1) and the $\mathrm{M} / \mathrm{M} / N / N$ queue (Section 4.2).

\subsection{The GI/GI/1 + GI queue}

The $\mathrm{M} / \mathrm{M} / 1+\mathrm{M}$ queueing model assumes that customers arrive according to a Poisson process with rate $\lambda$ to an infinite waiting room service facility, that their service times form an independent and identically distributed (i.i.d.) sequence of exponential random variables having mean $1 / \mu>0$, and that each customer independently reneges if his/her service has not begun within an exponentially distributed amount of time that has mean $1 / \gamma>0$. Theorem 2 of [18] supports approximating the number-in-system process $Q=\{Q(t), t \geq 0\}$ by a $(\sqrt{2 \lambda}, \lambda-\mu, \gamma)$ reflected OU process $Z$.

The more general GI/GI/1 + GI queueing model assumes that the customer arrival process is a renewal process with rate $\lambda$, that the service time distribution is general with mean $1 / \mu$, and that each customer independently reneges if his/her service has not begun within an amount of time that is distributed according to some probability distribution function $F$. In the case that $F$ has a density and $F^{\prime}(0)>0$ is finite, Theorem 3 of [20] combined with the arguments in the proof of Theorem 2 of [18] shows that $Q$ may be approximated by a $\left(\sqrt{2 \lambda}, \lambda-\mu, F^{\prime}(0)\right)$ reflected OU process. Note that this is consistent with the approximation for $Q$ in the previous paragraph since the value of the density of an exponential random variable at 0 is equal to its rate.

Our results in Section 3 (specifically, Proposition 3.2 and (3.5)) then imply for the $M / M / 1+$ M case that

$$
\mathbb{P}(Q(t) \geq x) \approx \mathbb{P}(Z(t) \geq x)=\int_{0}^{t} p_{(\lambda-\mu-\gamma x) / \gamma \sqrt{2 \lambda} \rightarrow(\lambda-\mu) / \gamma \sqrt{2 \lambda}}^{(1,0,-\gamma)}(s) \mathrm{d} s,
$$




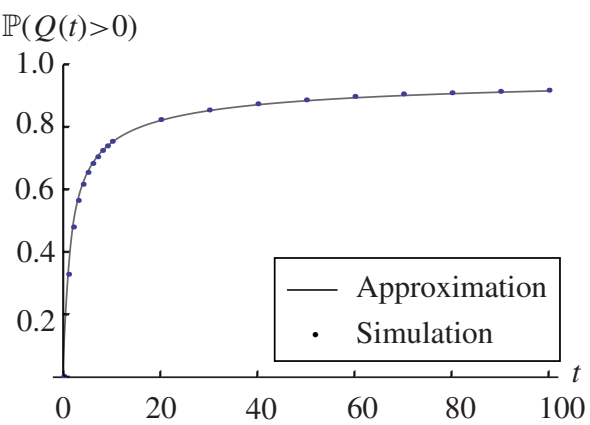

(a) $\mathbb{P}(Q(t) \geq 1), \gamma=0.001$

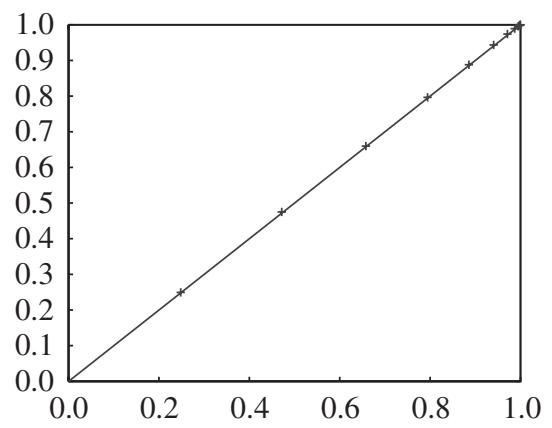

(c) P-P plot for $\mathbb{P}(Q(10)<x), \gamma=0.001$

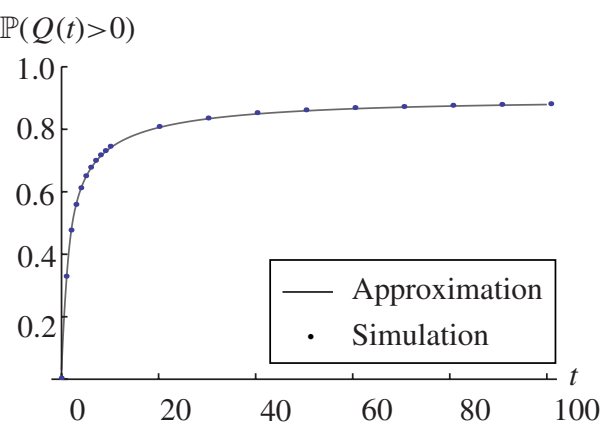

(b) $\mathbb{P}(Q(t) \geq 1), \gamma=0.01$

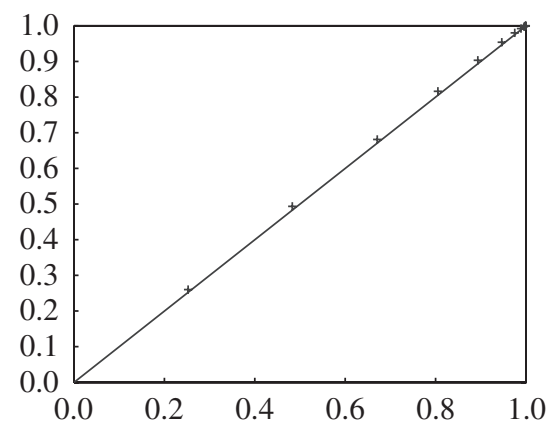

(d) P-P plot for $\mathbb{P}(Q(10)<x), \gamma=0.01$

Figure 1: Simulated and approximated results for the $\mathrm{M} / \mathrm{M} / 1+\mathrm{M}$ queueing model when $\lambda=\mu=0.5$, $\gamma=0.001$, so that $P_{R}=3.41 \%$, and $\lambda=\mu=0.5, \gamma=0.01$, so that $P_{R}=9.79 \%$. $P_{R}$ is the steady-state percentage of arriving customers that renege.

when $Q(0)=0$. For the GI/GI/1 + GI case, we may replace $\gamma$ with $F^{\prime}(0)$ in the above. Hence, we have an approximation for the transient distribution for the number-in-system process in a GI/GI/1 + GI queue. Note that the theory in [18] and [20] suggests that the approximation in (4.1) will be good when $\lambda$ and $\mu$ are close, and when $\gamma$ is small compared to $\lambda$ and $\mu$ (that is, the percentage of customers reneging is not too large). For related work, we refer the interested reader to Fralix [7], who derived the time-dependent moments of an $\mathrm{M} / \mathrm{M} / 1+\mathrm{M}$ queue, and then used those to obtain the time-dependent moment expressions for a reflected OU process.

We now proceed to verify approximation (4.1) in an $M / M / 1+M$ model via simulation. Note that even in the case of an $\mathrm{M} / \mathrm{M} / 1+\mathrm{M}$ model, the problem of finding an exact expression for its transient distribution appears to be very difficult (as is suggested by the computations in [21], which provide some performance measure expressions in terms of transforms for a many-server model with reneging). The plots in Figure 1 reveal that approximation (4.1) is very accurate, both for calculating the probability that the system is nonempty for a range of $t$ values, and for finding the entire distribution of $Q(t)$ for a fixed $t$. The simulation results shown are averaged over 10000 runs, stopped at the relevant time value. Note that we chose $\lambda=\mu$ so that we could use the very simple expression (3.4) when computing $\mathbb{P}(Z(t) \geq x)$. When $\lambda \neq \mu$, there is another source of error that comes into approximation (4.1) that is due to the methodology in [1] for computing the hitting time density function of an OU process. 


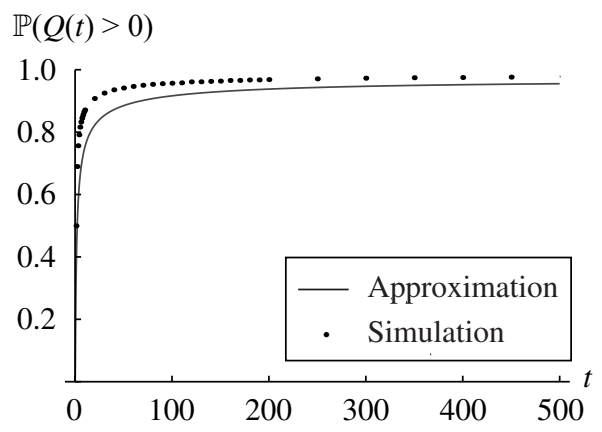

(a) $\mathbb{P}(Q(t) \geq 1)$

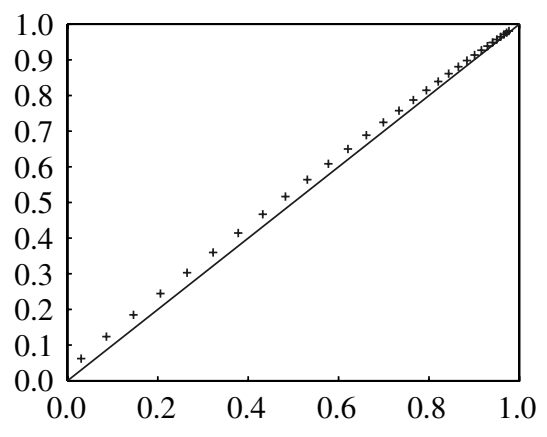

(c) P-P plot for $\mathbb{P}(Q(200)<x)$

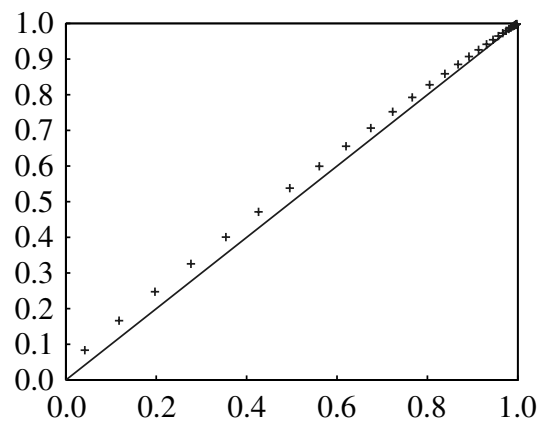

(b) P-P plot for $\mathbb{P}(Q(100)<x)$

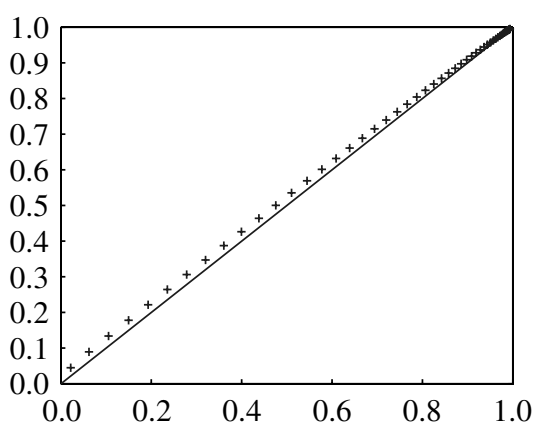

(d) P-P plot for $\mathbb{P}(Q(500)<x)$

FIGURE 2: Simulated and approximated results for the GI/GI/1 + GI queueing model when the interarrival and service time distributions are gamma $(2,2)$ and the reneging distribution $F$ is uniform on $[0,1000]$.

The plots in Figure 2 verify approximation (4.1) in a GI/GI/1 + GI queueing model. Note that the relevant approximating reflected OU process is exactly the same as in the $M / M / 1+M$ queueing model in Figure 1(a) and (c). We observe that the transient distribution approximation is good for 'medium' $t$ but not for 'small' $t$. (The simulation results in [20] imply that the approximation is good for 'large' $t$, when the system is close to its steady state.) The GI/GI/1+ GI queue that we simulated had simulated steady-state mean number-in-system 18.12 , and simulated mean number-in-system at times $t=100, t=200$, and $t=500$ of 7.73, 10.43, and 14.43, respectively. Then, the displayed P-P plots for $\mathbb{P}(Q(t)<x)$ in Figure 2 are such that the transient distribution is relevant (and not the steady-state distribution).

\subsection{The $\mathrm{M} / \mathrm{M} / N / N$ queue}

The $\mathrm{M} / \mathrm{M} / N / N$ queueing model assumes that customers arrive at rate $\lambda>0$ in accordance with a Poisson process to a service facility with $N$ servers and no additional place for waiting, and that their service times form an i.i.d. sequence of exponential random variables with mean $1 / \mu$. Any arriving customer that finds $N$ customers in the system is blocked from receiving service, and so is lost. Suppose that we let the number of servers in the system be a function of the arrival rate $\lambda$, and assume that

$$
N^{\lambda}=\frac{\lambda+\beta \sqrt{\lambda}}{\mu} \text { for } \beta \in \mathbb{R} .
$$




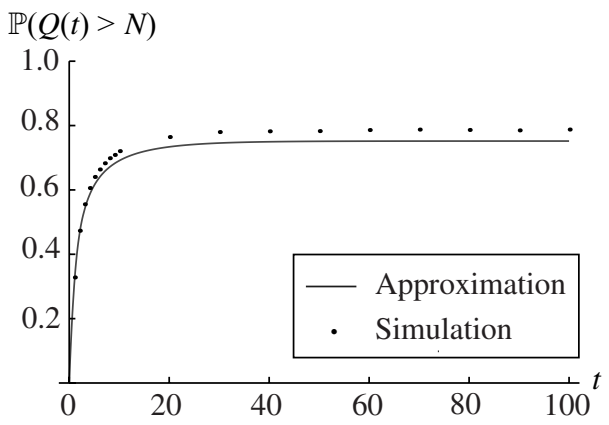

(a) $\mathbb{P}(Q(t)<N), \lambda=0.5, \mu=0.05$, and $N=10$

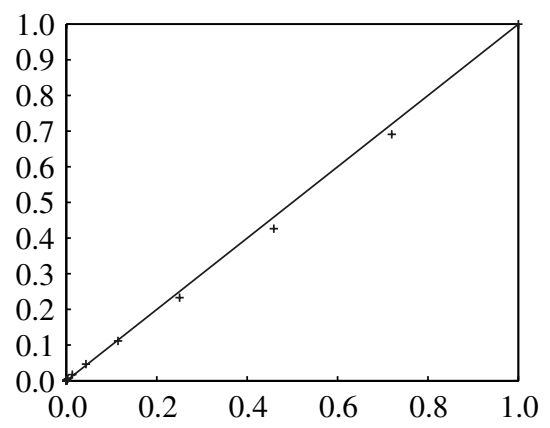

(c) P-P plot for $\mathbb{P}(Q(10) \leq x), \lambda=0.5$, $\mu=0.05$, and $N=10$

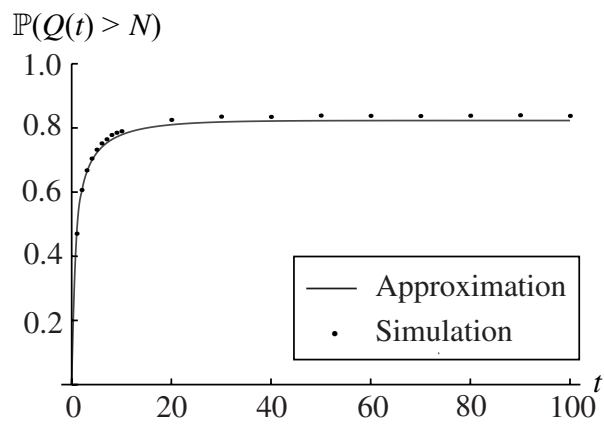

(b) $\mathbb{P}(Q(t)<N), \lambda=1, \mu=0.05$, and $N=20$

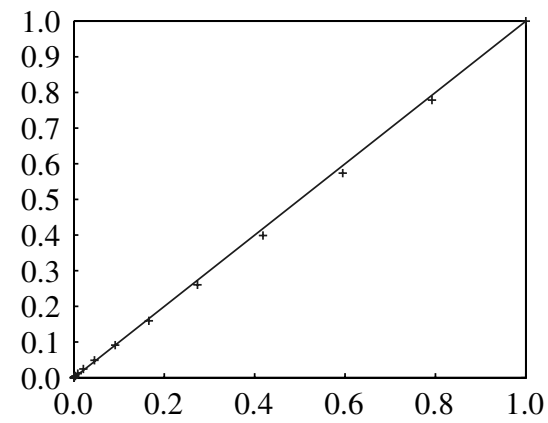

(d) P-P plot for $\mathbb{P}(Q(10) \leq x), \lambda=1$, $\mu=0.05$, and $N=20$

FIGURE 3: Simulated and approximated results for the $\mathrm{M} / \mathrm{M} / N / N$ queueing model.

Then, Srikant and Whitt [16] showed that

$$
\frac{N^{\lambda}-Q^{\lambda}}{\sqrt{\lambda}} \Rightarrow Z \quad \text { as } \lambda \rightarrow \infty,
$$

where $Z$ is a $(\sqrt{2}, \beta, \mu)$ reflected OU process. Hence, our results in Section 3 (specifically, Proposition 3.2 and (3.5)) imply that

$$
\begin{aligned}
\mathbb{P}(Q(t) \leq x) & =\mathbb{P}\left(\frac{N-Q(t)}{\sqrt{\lambda}} \geq \frac{N-x}{\sqrt{\lambda}}\right) \\
& \approx \mathbb{P}\left(Z(t) \geq \frac{N-x}{\sqrt{\lambda}}\right) \\
& =\int_{0}^{t} p_{(N-2 \lambda / \mu+x) / 2 \sqrt{\lambda} \rightarrow(\mu N-\lambda) / \sqrt{\lambda}}^{(1,0,-\mu)}(s) \mathrm{d} s,
\end{aligned}
$$

when $Q(0)=N$.

In Figure 3 we compare simulated results for the $\mathrm{M} / \mathrm{M} / N / N$ queue to values obtained using the approximation in (4.3). We see that the approximation becomes more accurate as $N$ becomes larger, which is as expected. Note that, by (4.2), this also implies that the utilization 
is close to 1 . The simulation results shown are the average over 10000 runs, stopped at the relevant time value.

\section{References}

[1] Alili, L., Patie, P. and Pedersen, J. L. (2010). Representations of the first hitting time density of an OrnsteinUhlenbeck process. Preprint.

[2] Anantharam, V. and Konstantopoulos, T. (2011). Integral representation of Skorokhod reflection. Proc. Amer. Math. Soc. 139, 2227-2237.

[3] Burdzy, K., Kang, W. and Ramanan, K. (2009). The Skorokhod problem in a time-dependent interval. Stoch. Process. Appl. 119, 428-452.

[4] Chaleyat-Maurel, M., El Karoui, N. and Marchal, B. (1980). Reflexion discontinue et systèmes stochastiques. Ann. Prob. 8, 1049-1067.

[5] Cox, J. T. ANd Rosler, U. (1984). A duality relation for entrance and exit laws for Markov processes. Stoch. Process. Appl. 16, 141-156.

[6] DupuIs, P. AND IsHII, H. (1991). On Lipschitz continuity of the solution mapping to the Skorokhod problem, with applications. Stoch. Stoch. Reports 35, 31-62.

[7] Fralix, B. H. (2012). On the time-dependent moments of Markovian queues with reneging. Queueing Systems 20pp.

[8] Harrison, J. M. and Reiman, M. I. (1981). Reflected Brownian motion on an orthant. Ann. Prob. 9, 302-308.

[9] Kruk, Ł., Lehoczky, J., Ramanan, K. and Shreve, S. (2007). An explicit formula for the Skorokhod map on [0, a]. Ann. Prob. 35, 1740-1768.

[10] Kruk, Ł., Lehoczky, J., Ramanan, K. and Shreve, S. (2008). Double Skorokhod map and reneging real-time queues. In Markov Processes and Related Topics: A Festschrift for Thomas G. Kurtz (Inst. Math. Statist. Collect. 4), Institute of Mathematical Statistics, Beachwood, OH, pp. 169-193.

[11] LinetsKy, V. (2004). Computing hitting time densities for OU and CIR processes: applications to mean-reverting models. J. Comput. Finance 7, 1-22.

[12] Pitman, J. And Yor, M. (1981). Bessel processes and infinitely divisible laws. In Stochastic Integrals (Lecture Notes Math. 851), Springer, Berlin, pp. 285-370.

[13] Ramanan, K. (2006). Reflected diffusions via the extended Skorokhod map. Electron. J. Prob. 36, 934-992.

[14] Sigman, K. and Ryan, R. (2000). Continuous-time monotone stochastic recursions and duality. Adv. Appl. Prob. 32, 426-445.

[15] Skorokhod, A. V. (1961). Stochastic equations for diffusions in a bounded region. Theoret. Prob. Appl. 6, 264-274.

[16] Srikant, R. and Whitt, W. (1996). Simulation run lengths to estimate blocking probabilities. ACM Trans. Model. Comput. Simul. 6, 7-52.

[17] TanaKa, H. (1979). Stochastic differential equations with reflecting boundary conditions in convex regions. Hiroshima Math J. 9, 163-177.

[18] Ward, A. R. AND Glynn, P. W. (2003). A diffusion approximation for a Markovian queue with reneging. Queueing Systems 43, 103-128.

[19] Ward, A. R. AND Glynn, P. W. (2003). Properties of the reflected Ornstein-Uhlenbeck process. Queueing Systems 44, 109-123.

[20] WARD, A. R. AND GLYNN, P. W. (2005). A diffusion approximation for a GI/GI/1 queue with balking or reneging. Queueing Systems 50, 371-400.

[21] Whiтt, W. (1999). Improving service by informing customers about anticipated delays. Manag. Sci. 45, 192207.

[22] Zhang, T. S. (1994). On the strong solutions of one-dimensional stochastic differential equations with reflecting boundary. Stoch. Process. Appl. 50, 135-147. 